\title{
Prostaglandin analog effects on cerebrospinal fluid reabsorption via nasal mucosa
}

\section{Short title: Prostaglandins and CSF absorption}

\author{
Michelle G. Pedler ${ }^{1}$, J. Mark Petrash ${ }^{1}$, and Prem S. Subramanian ${ }^{1,2,3,4}$ \\ Departments of ${ }^{1}$ Ophthalmology, ${ }^{2}$ Neurology, and ${ }^{3}$ Neurosurgery, University of Colorado School \\ of Medicine, Aurora, CO, USA and ${ }^{4}$ Division of Ophthalmology, Uniformed Services University of \\ the Health Sciences, Bethesda, MD, USA
}

Corresponding author:

Prem S. Subramanian, MD, PhD

University of Colorado Sue Anschutz-Rodgers Eye Center

1675 Aurora Ct Mail Stop F731

Aurora CO 80045 USA

$+1.720 .848 .2500$

$+1.720-848.5014$ fax

prem.subramanian@cuanschutz.edu 


\begin{abstract}
Introduction: Cerebrospinal fluid (CSF) outflow has been demonstrated along nasal lymphatics via olfactory nerve projections; flow may be increased by stimulating lymphatic contractility using agents such as noradrenaline and the thromboxane A2 analog U46619. Lymphatics elsewhere in the body show increased contractility upon exposure to the prostaglandin F2alpha analog isoprostane-8-epi-prostaglandin. We investigated the ability of ophthalmic prostaglandin F2alpha analogs to increase CSF outflow when applied to the nasal mucosa by inhalation.
\end{abstract}

Methods: Latanoprost $(0.1,0.5$, or $1 \mathrm{mg} / \mathrm{ml})$, bimatoprost $(0.3$ or $3 \mathrm{mg} / \mathrm{ml})$, travoprost $(0.04$ or $0.4 \mathrm{mg} / \mathrm{ml})$, latanoprostene bunod $(0.24$ or $2.4 \mathrm{mg} / \mathrm{ml})$, tafluprost $(0.25$ or $2.5 \mathrm{mg} / \mathrm{ml})$, or vehicle (10\% DMSO) was administered to awake adult C57B/6 mice by nasal inhalation of $2 \mu$ l droplets. A total of 67 animals were studied including controls. General anesthesia was induced by injection, and fluorescent tracer (AlexaFluor647-labelled ovalbumin) was injected under stereotaxic guidance into the right lateral ventricle. Nasal turbinate tissue was harvested and homogenized after 1 hour for tracer detection by ELISA and fluorometric analysis.

Results: Inhalation of latanoprost $0.5 \mathrm{mg} / \mathrm{ml}$ and $1 \mathrm{mg} / \mathrm{ml}$ led to a 11.5 -fold increase in tracer recovery from nasal turbinate tissues compared to controls $(3312 \mathrm{pg} / \mathrm{ml}$ vs $288 \mathrm{pg} / \mathrm{ml}, \mathrm{p}<0.001$ for $0.5 \mathrm{mg} / \mathrm{ml} ; 3355 \mathrm{pg} / \mathrm{ml}$ vs $288 \mathrm{pg} / \mathrm{ml}, \mathrm{p}<0.001$ for $1 \mathrm{mg} / \mathrm{ml}$ ), while latanoprost $0.1 \mathrm{mg} / \mathrm{ml}$ enhanced recovery 6 -fold $(1713 \mathrm{pg} / \mathrm{ml}$ vs $288 \mathrm{pg} / \mathrm{ml}, \mathrm{p}<0.01)$. Tafluprost $0.25 \mathrm{mg} / \mathrm{ml}$ and 
bimatoprost $0.3 \mathrm{mg} / \mathrm{ml}$ showed a modest $(1.4 \mathrm{x}, \mathrm{p}<0.05)$ effect, and the remaining agents showed no significant effect on tracer recovery.

Conclusions: Prostaglandin F2alpha analogs delivered by nasal inhalation resulted in increased nasal recovery of a CSF fluorescent tracer, implying increased CSF outflow via the nasal lymphatics. The greatest effect, partially dose-dependent, was observed using latanoprost. Further studies are needed to determine the efficacy of these agents in reducing ICP in short and long-term applications. 


\section{Introduction}

Cerebrospinal fluid (CSF) is produced in the lateral ventricles by active transport across the cell membranes of epithelial cells lining the arachnoid villi and is dependent upon $\mathrm{Na} / \mathrm{K}$ ion channel activity(1). CSF then flows freely through the ventricles, fills several cisterns as well as sulci along the surface of the brain, and is maintained in homeostasis via reabsorption through several putative pathways(1), including via arachnoid granulations(2). CSF also is present in the meningeal coverings of the cranial nerves that exist before they exit the cranial compartment(2), and CSF also can flow along the most proximal portion of spinal root ganglia and nerves(3). Studies demonstrate that normal CSF outflow occurs along these nerves and nerve roots, although the relative importance of each pathway in the maintenance of normal CSF turnover remains controversial(1). CSF movement along the olfactory nerves through the cribriform plate and into the nasal lymphatic system comprises a major outflow pathway in experimental animals, and it may take on greater importance when intracranial pressure (ICP) is elevated(4-7). Because extracranial lymphatic outflow is not dependent upon venous sinus pressures, CSF flow along cranial nerves may not be affected when cerebral venous outflow is diminished, and increased CSF flow may compensate for reduced absorption via the arachnoid granulations(8).

Current treatment strategies for disorders of elevated ICP use medications to reduce CSF production by inhibition of carbonic anhydrase (acetazolamide, methazolamide), diuresis (same agents as well as furosemide), and/or sodium transport inhibition (furosemide)(9). No medications are available that will increase CSF outflow, and the olfactory lymphatics provide an accessible target for such a treatment(10). Although lymphatic channels are often thought to 
carry fluid passively into the venous system, increased lymphatic contractility can be induced pharmacologically. Experiments with lower extremity lymphatic stimulation have demonstrated improvement in peripheral edema with use of a variety of pharmacologic agents, such as the prostaglandin F2 alpha analogue isoprostane 8-epi-prostaglandin F2alpha (PGF2alpha)(11). CSF pressure monitoring in sheep after intranasal delivery of pharmacologic agents that may affect lymphatic contractility suggests that CSF outflow facility may be manipulated in this manner(10). We therefore hypothesized that commercially available PGF2alpha analogues, widely used in the treatment of open angle glaucoma, might increase CSF outflow through the nasal lymphatics in an animal model and sought to directly demonstrate this effect.

\section{Methods}

Animal use protocols were approved by the Institutional Animal Care and Use Committee (IACUC) of the University of Colorado School of Medicine. Commercially available PGF2-alpha analogues used in the treatment of glaucoma were selected for evaluation; these included latanoprost, bimatoprost, travoprost, latanoprostene bunod, and tafluprost. Initial experiments were conducted with topical ophthalmic preparations, and pharmaceutical grade drug also was obtained and placed into solution for use in a more concentrated form. Agents were solubilized in DMSO and diluted in normal saline.

Awake adult male and female C57/BL6 mice (approximately 6 weeks of age) were administered intranasal vehicle (10\% DMSO) or drug solution (total $8 \mu \mathrm{l})$ by placing droplets ( 2 $\mu l)$ of liquid at the nares using a micropipette and allowing the animal to inhale the liquid spontaneously(12). Anesthesia with ketamine and xylazine then was administered via 
intraperitoneal injection, and anesthetized animals were placed within a stereotaxic apparatus. A midline incision was created along the scalp, and the right lateral ventricle was targeted with placement of a 26 ga needle to a depth of $3 \mathrm{~mm}$ into the brain at a position $1 \mathrm{~mm}$ lateral and $0.3 \mathrm{~mm}$ anterior to bregma(13). A $5 \mu \mathrm{l}$ intraventricular injection of fluorescent tracer (AlexaFluor ${ }^{647}$-ovalbumin solution) was delivered at a rate of $100 \mathrm{nl} / \mathrm{sec}$ using a Hamilton syringe and an electric pump. Animals were allowed to recover from the procedure and then sacrificed 60 minutes post-injection. Nasal turbinates were harvested, and tissue extracts were analyzed by ELISA and fluorometrically.

Statistical analyses were conducted with Stata (College Station, TX) with both two-tailed Student's t-tests and ANOVA RM with Dunnet's post hoc analysis.

\section{Results}

The experimental technique was validated by assessing anatomic placement of the tracer into the lateral ventricle (Fig. 1) and by recovery of fluorescent tracer from nasal mucosa after ventricular injection in the absence of intranasal treatment. Timepoints for tissue harvest were selected based on increased recovery at 60 min versus $30 \mathrm{~min}$, with later harvest raising concern for potential contamination of specimens by hematogenous CSF absorption of tracer and recirculation throughout the tissues via arterial flow. Other tissues (spleen, liver) from injected animals did not demonstrate significant levels of ovalbumin tracer relative to uninjected animals after 60 minutes.

Figure 1. Evaluation of fluorescent tracer injection. A. Photomicrograph (20X magnification) of brain harvested after stereotaxic injection of labelled ovalbumin into the right lateral ventricle 
showing localized signal within the ventricle and along the injection track. B. Section from a control, uninjected animal demonstrating no staining in the ventricle.

A total of 67 animals were studied using a scaled dosing technique in which each prostaglandin analog was evaluated at its commercial ophthalmic concentration as well as one or two more concentrated levels (either $5 x$ or $10 x$ ). When inhaled in their commercial ophthalmic formulations, both bimatoprost and tafluprost showed an approximately 1.5 fold increase in nasal tracer recovery relative to vehicle-treated controls (Fig. 2, p<0.05). Neither drug showed a significant effect when used at a higher concentration; additionally, neither travoprost nor latanoprostene bunod demonstrated the ability to increase nasal tracer recovery above control levels at any concentration used (Fig. 2). Intranasal delivery of latanoprost resulted in a semi dose-dependent increase in tracer recovery from the nasal mucosa after 60 min, with approximately 10 -fold increased levels observed relative to control animals when 0.5 $\mathrm{mg} / \mathrm{ml}$ or $1 \mathrm{mg} / \mathrm{ml}$ solution was used (Fig. 3, $\mathrm{p}<0.001$ compared to control). The standard ophthalmic dose, $0.1 \mathrm{mg} / \mathrm{ml}$, resulted in approximately 6-fold increased tracer recovery (Fig. 3, $\mathrm{p}<0.01$ vs control).

Figure 2. Fluorometric analysis of nasal turbinate homogenates after prostaglandin F2alpha agonist bimatoprost, latanoprostene bunod, tafluprost, and travoprost inhalation and ventricular tracer injection. After awake inhalation of drug or vehicle (10\% DMSO), animals underwent stereotaxic right ventricular injection of fluorescent-labelled tracer. Nasal turbinates were harvested 60 min post-injection. Asterisks indicate $p<0.05$ relative to controls. 
Figure 3. Comparison of the effect of latanoprost intranasal application to other PGF2alpha analogues in the recovery from nasal turbinates of fluorescently tagged ovalbumin after CSF injection. Experimental conditions were identical to Figure $2 ; * *=p<0.05, * * *=p<0.01$.

\section{Discussion}

In this study, latanoprost showed a dose-dependent ability to increase recovery of a CSF-based fluorescent tracer substance from the nasal mucosa of experimental animals shortly after intranasal inhalation. A lesser effect was observed with tafluprost and bimatoprost, and these drugs did not appear to have a dose-dependent effect. Two additional prostaglandin analogues, latanoprostene bunod and travoprost, showed no apparent impact on tracer recovery when inhaled intranasally.

Prior work with pharmacologic agents introduced into the nasal cavities of sheep by nebulization showed an increase in CSF outflow as measured by an intracranial pressure monitoring system by which fluid was infused into the lateral ventricle and the resulting pressure changes assessed(10). In this system, it was calculated that a 2.29 -fold to 2.44 fold increase in CSF outflow was achieved with the use of either NG-monomethyl L-arginine or U46619, a thromboxane A2 analogue. Interestingly, while the latter agent increases lymphatic contractility, the former inhibits nitric oxide synthesis and causes smooth muscle relaxation, and the authors postulated that an increased calibre of the lymphatic channels might allow greater passive flow along a pressure gradient in some instances(10).

The relative contribution of nasal lymphatic drainage to overall CSF outflow has not been determined in humans. In both sheep and rat models, injection of a radiolabelled tracer 
into the CSF with recovery from lymphatic and other sources suggests that at least $50 \%$ of normal CSF drainage in awake animals may occur via the lymphatic system(14,15). Additionally, induced communicating hydrocephalus in a rodent model is associated with reduced CSF lymphatic outflow along the olfactory and nasal mucosal pathways(6).

We observed that latanoprost had a stronger effect on CSF tracer recovery than did other prostaglandin F2alpha analogues. Prior studies have shown a variable effect of different pharmacologic agents of the same class on vascular contractility. The isoprostane 8-epiPGF2alpha has a more potent effect on lymphatic channels than does U46619(11), and application of U46619, PGF2alpha itself, and latanoprost had a significantly greater effect on porcine ciliary artery constriction than did travoprost(16). Latanoprost also causes increased lymphatic outflow from the eye in mice(17), and a diminished effect of latanoprost on intraocular pressure has been observed in humans who have undergone surgical ligation of cervical lymphatics for cancer treatment(18). However, the relative effect of other PGF2alpha analogues on ocular lymphatic drainage has not been reported.

In conclusion, we found that a single nasal inhalation of the PGF2alpha analogue latanoprost resulted in an increased recovery of a CSF tracer from nasal mucosa, indicating greater passage of CSF through olfactory lymphatic channels, with a weaker effect seen with bimatoprost and tafluprost. We did not evaluate the longevity of this effect after a single dose, nor did we determine if repeated or chronic dosing would enhance tracer recovery. Further study is needed to assess the local effect on nasal mucosa from acute and chronic application of latanoprost solutions and to determine its tolerability. Based on ocular studies(17), we anticipate that lymphatic activity induced by latanoprost would persist and not diminish over 
time. If the drug can be used intranasally on a chronic basis, it may be a useful therapeutic agent for humans with disorders of ICP elevation.

Acknowledgements: none

\section{References}

1. Bothwell SW, Janigro D, Patabendige A. Cerebrospinal fluid dynamics and intracranial pressure elevation in neurological diseases. Fluids Barriers Cns. 2019;16(1):9.

2. Tsutsumi S, Ogino I, Miyajima M, Nakamura M, Yasumoto Y, Arai H, et al. Cranial Arachnoid Protrusions and Contiguous Diploic Veins in CSF Drainage. Am J Neuroradiol [Internet]. 2014 Sep;35(9):1735-9. Available from:

http://eutils.ncbi.nlm.nih.gov/entrez/eutils/elink.fcgi?dbfrom=pubmed\&id=24948506\&retmod $\mathrm{e}=\mathrm{ref} \& \mathrm{cmd}=$ prlinks

3. Pahlavian SH, Yiallourou T, Tubbs RS, Bunck AC, Loth F, Goodin M, et al. The Impact of Spinal Cord Nerve Roots and Denticulate Ligaments on Cerebrospinal Fluid Dynamics in the Cervical Spine. Plos One. 2014;9(4):e91888.

4. Kida S, Pantazis A, Weller RO. CSF drains directly from the subarachnoid space into nasal lymphatics in the rat. Anatomy, histology and immunological significance. Neuropath Appl Neuro. 1993;19(6):480-8.

5. Boulton M, Young A, Hay J, Armstrong D, Flessner M, Schwartz M, et al. Drainage of CSF through lymphatic pathways and arachnoid villi in sheep: measurement of $125 \mathrm{l}$-albumin clearance. Neuropathology and applied neurobiology. 1996 Aug;22(4):325 333.

6. Nagra G, Li J, McAllister JP, Miller J, Wagshul M, Johnston M. Impaired lymphatic cerebrospinal fluid absorption in a rat model of kaolin-induced communicating hydrocephalus. American journal of physiology Regulatory, integrative and comparative physiology. 2008;294(5):R1752-9.

7. Ahn JH, Cho H, Kim J-H, Kim SH, Ham J-S, Park I, et al. Meningeal lymphatic vessels at the skull base drain cerebrospinal fluid. Nature. 2019;572(7767):62-6.

8. Koh L, Nagra G, Johnston M. Properties of the Lymphatic Cerebrospinal Fluid Transport System in the Rat: Impact of Elevated Intracranial Pressure. J Vasc Res. 2007;44(5):423-32. 
9. Dave SB, Subramanian PS. Pseudotumor cerebri: An update on treatment options. Indian J Ophthalmol. 2014 Oct;62(10):996-8.

10. Kim H, Moore SA, Johnston MG. Potential for intranasal drug delivery to alter cerebrospinal fluid outflow via the nasal turbinate lymphatics. Fluids Barriers Cns. 2014 Feb 15;11(1):4.

11. Sinzinger $\mathrm{H}$, Oguogho A, Kaliman J. Isoprostane 8-epi-prostaglandin F2 alpha is a potent contractor of human peripheral lymphatics. Lymphology. 1997;30(3):155-9.

12. Hanson LR, Fine JM, Svitak AL, Faltesek KA. Intranasal Administration of CNS Therapeutics to Awake Mice. J Vis Exp. 2013;(74).

13. DeVos SL, Miller TM. Direct Intraventricular Delivery of Drugs to the Rodent Central Nervous System. J Vis Exp Jove. 2013;(75):50326.

14. Boulton M, Flessner M, Armstrong D, Mohamed R, Hay J, Johnston M. Contribution of extracranial lymphatics and arachnoid villi to the clearance of a CSF tracer in the rat. Am J Physiology-regulatory Integr Comp Physiology. 1999;276(3):R818-23.

15. Boulton M, Flessner M, Armstrong D, Hay J, Johnston M. Lymphatic drainage of the CNS: effects of lymphatic diversion/ligation on CSF protein transport to plasma. Am J Physiologyregulatory Integr Comp Physiology. 1997;272(5):R1613-9.

16. Vysniauskiene I, Allemann R, Flammer J, Haefliger IO. Vasoactive Responses of U46619, PGF2 $\alpha$, Latanoprost, and Travoprost in Isolated Porcine Ciliary Arteries. Invest Ophth Vis Sci. 2006;47(1):295-8.

17. Tam ALC, Gupta N, Zhang Z, Yücel YH. Latanoprost Stimulates Ocular Lymphatic Drainage: An In Vivo Nanotracer Study. TransI Vis Sci Technology [Internet]. 2013 Aug;2(5):3-3. Available from: https://www.ncbi.nlm.nih.gov/pubmed/24049723

18. Kim YK, Na KI, Jeoung JW, Park KH. Intraocular Pressure-Lowering Effect of Latanoprost Is Hampered by Defective Cervical Lymphatic Drainage. Acott TS, editor. Plos One.

2017;12(1):e0169683. 

Figure 1B 


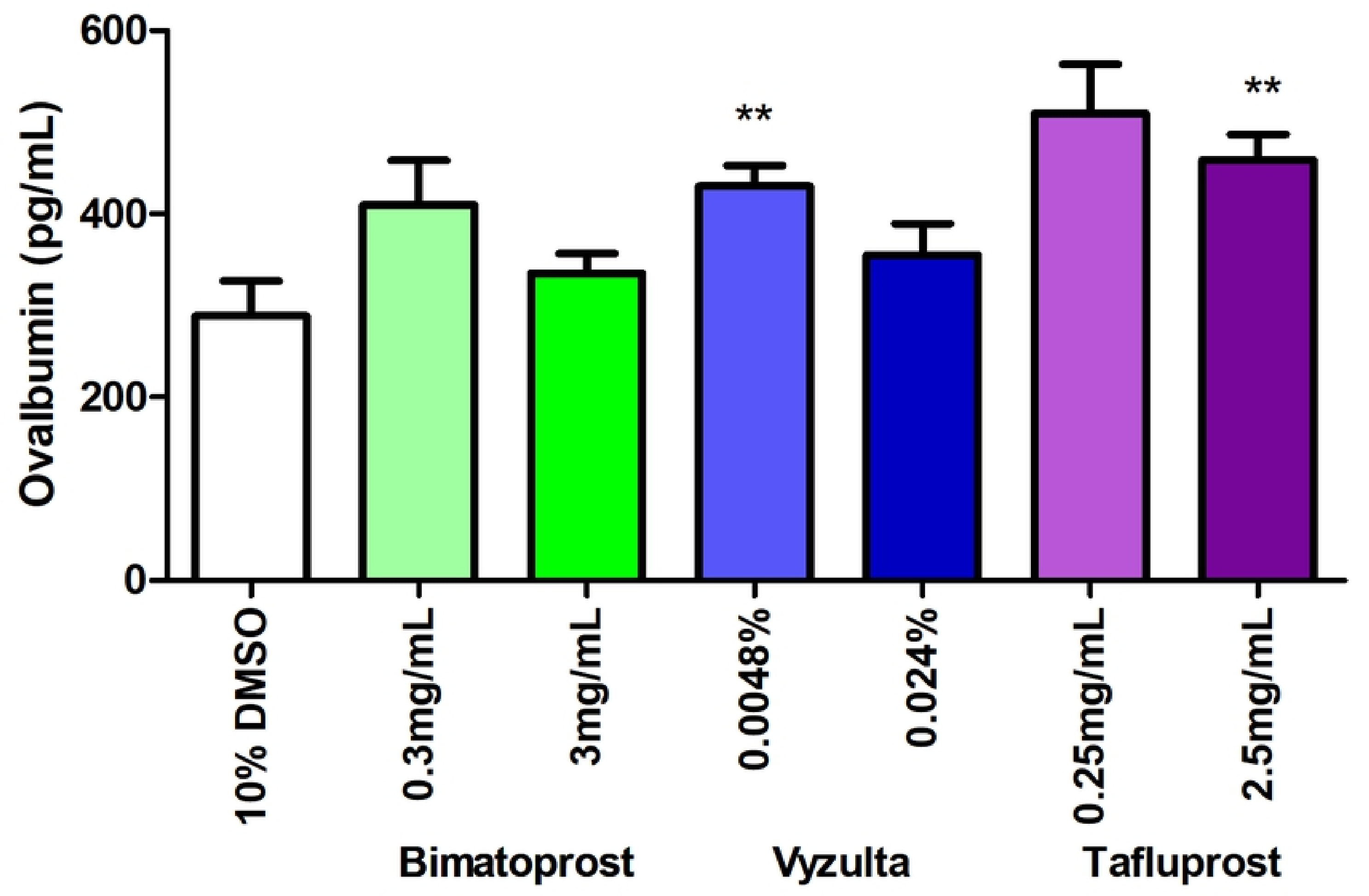

Figure 2 


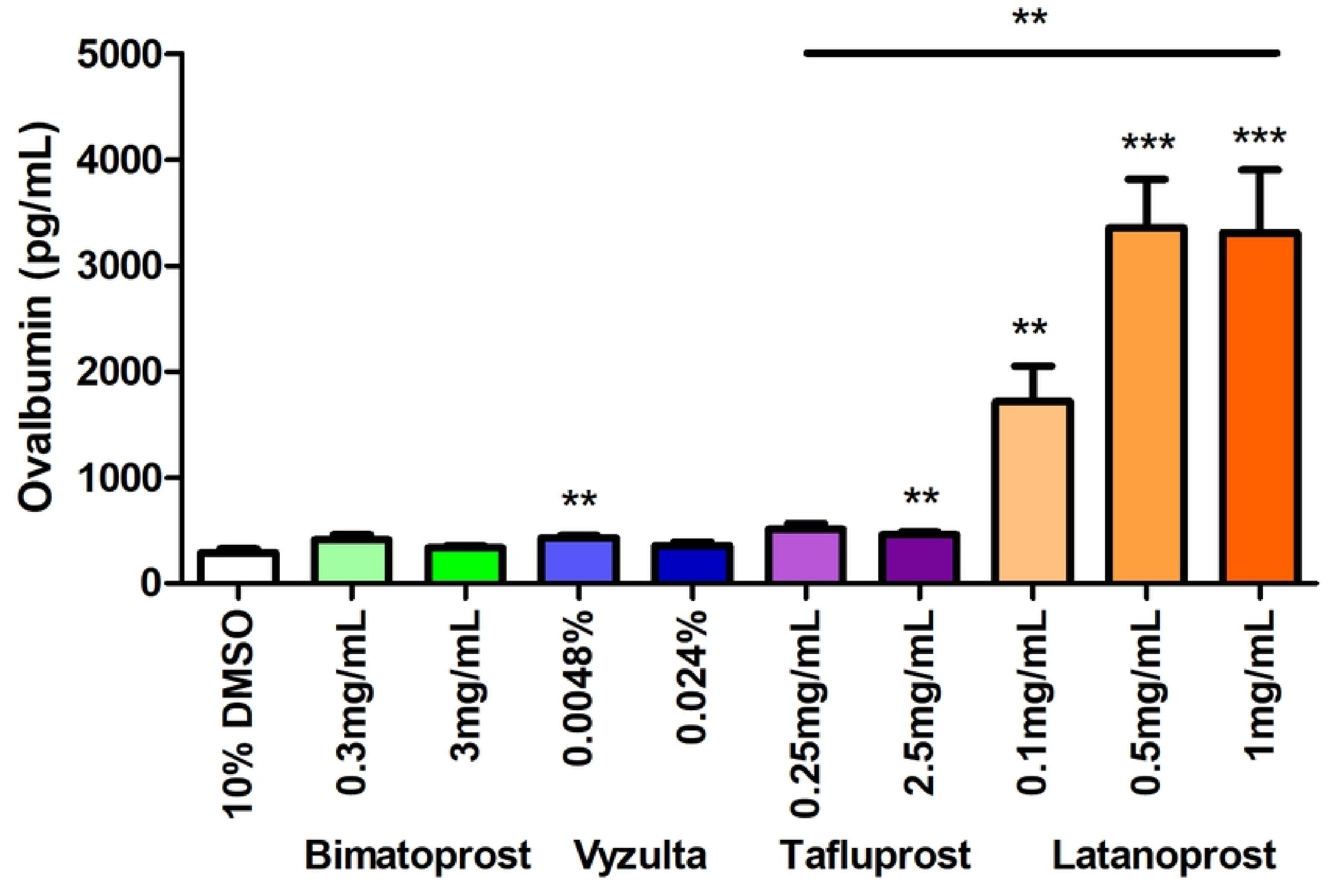

Figure 3 